\title{
THE PRESSFET: AN INTEGRATED ELECTRET-MOSFET BASED PRESSURE SENSOR
}

\section{J. A. VOORTHUYZEN and P. BERGVELD}

Twente University of Technology, P.O. Box 217, 7500 AE Enschede (The Netherlands)

(Received February 5, 1987; in revised form October 13, 1987; accepted December 14, 1987)

\section{Abstract}

We present a new MOSFET-based pressure sensor, incorporating an air gap that is a function of pressure and a permanently charged dielectric layer (electret) between the gate and bulk of the MOS structure. In this way we obtain a MOSFET with a pre-charged variable gate capacitance. The theory of this sensor and the NMOS-compatible process for its realization are given. We present also the first experimental results on this new sensor. We have determined the characteristics of sensors with outer dimensions of $1 \mathrm{~mm} \times$ $2 \mathrm{~mm} \times 0.3 \mathrm{~mm}$ and measured a maximum sensitivity of about $10 \mathrm{~mA} / \mathrm{A}$ / $100 \mathrm{~mm} \mathrm{Hg}$, which is about ten times higher than the sensitivity of piezoresistive pressure sensors with comparable dimensions. Drawbacks of the present sensor design are a relatively high temperature sensitivity and a rather complex fabrication process.

\section{Introduction}

In a recent paper [1] it has been shown that the integration of a field effect transistor and a permanently charged polymer foil (electret) offers a capacitive pressure sensor with a relatively high sensitivity. Although this sensor principle can be used for the realization of various types of micromechanical sensors, this paper describes the research on a pressure sensor.

An electret and a MOSFET structure can be combined in several ways. Using the detailed theoretical analysis already presented [2], we have decided to investigate the configuration as schematically drawn in Fig. 1.

As can be seen, it consists (from top to bottom) of a thin conductive diaphragm, an air gap, a polymer layer that contains electric charge, a silicon dioxide layer and a boron-doped silicon substrate with two heavily phosphorus-doped regions, the source and drain. The PRESSFET, as constructed in this way, can be regarded as a new type of FET device with a sandwich dielectric layer between the gate (the conductive diaphragm) and the silicon. The current in the channel (the region between the source and 


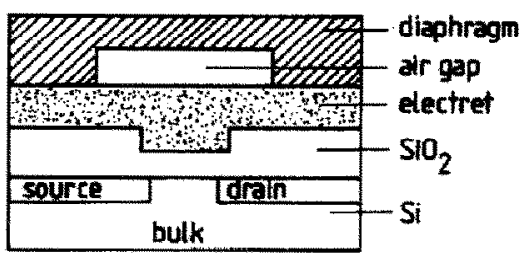

Fig. 1. Schematic drawing of the PRESSFET.

drain) depends on the value of the capacitance between the gate and silicon. Realizing that this capacitance is pressure sensitive, the drain current is thus a function of pressure.

In Section 2 the theoretical behaviour of the structure will be discussed. We will show that the device operation can be described by using the theory of the MOS transistor. In Section 3 the fabrication procedure will be explained step by step. In Section 4 the experimental results are presented, which are discussed in Section 5.

\section{Theoretical behaviour of the PRESSFET}

As mentioned above, the structure of Fig. 1 can be considered as a field effect transistor with a sandwich dielectric. The total capacitance $C_{t}$ per unit area of this layered dielectric can easily be written as a function of the unit area capacitances $C_{\mathrm{a}}$ of the air gap, $C_{\mathrm{e}}$ of the electret foil and $C_{\mathrm{ox}}$ of the silicon dioxide:

$$
C_{\mathrm{t}}=\frac{C_{\mathrm{a}} C_{\mathrm{e}} C_{\mathrm{ox}}}{C_{\mathrm{a}} C_{\mathrm{e}}+C_{\mathrm{a}} C_{\mathrm{ox}}+C_{\mathrm{e}} C_{\mathrm{ox}}}
$$

Note that the unit area capacitance of an insulating layer $i$ can be written as $C_{i}=\epsilon_{0} \epsilon_{i} / d_{i}$, with $\epsilon_{0}$ the dielectric constant of vacuum, $\epsilon_{\mathrm{i}}$ the relative dielectric constant and $d_{i}$ the thickness of the layer.

The values of the capacitances of the electret and the oxide layer are fixed, but the capacitance of the air gap will be a function of pressure due to the deflection of the thin conductive diaphragm.

Generally the deflection of a thin circular diaphragm is a function of the radial distance from its centre. Assuming the dimensions of the channel of the transistor to be small as compared to the dimensions of the diaphragm, its displacement can be regarded to be uniform and equal to the deflection at the centre. If the value of the air-gap space for zero pressure is written as $d_{\mathrm{n} 0}$, the actual value $d_{\mathrm{a}}$ can be written as [3]:

$d_{\mathrm{a}}=d_{\mathrm{a} 0}-\frac{P a^{4}}{64 D}$

with $P$ the pressure, $a$ the radius of the diaphragm and $D$ the so-called flexural rigidity [3]. 
Considering this equation, it will be clear that the maximum pressure $P_{m}$ that can be detected by the sensor is the pressure for which $d_{\mathbf{a}}$ becomes equal to zero. This means that $\boldsymbol{P}_{\mathrm{m}}$ can be written as

$$
P_{m}=\frac{64 D d_{\mathrm{aO}}}{a^{4}}
$$

In describing the drain current of the PRESSFET, we can use the description for the drain current of a MOSFET, as given in the literature [4]. Note that in doing so we have to replace the oxide capacitance by the total capacitance $C_{\mathrm{t}}$ per unit area. Assuming the PRESSFET to be biased in the so-called unsaturated mode, we obtain:

$I_{\mathrm{d}}=\mu \frac{W}{L} C_{\mathrm{t}}\left[\left(V_{\mathrm{ts}}-V_{\mathrm{t}}\right) V_{\mathrm{d} s}-0.5 V_{\mathrm{ds}}{ }^{2}\right]$

with $\mu$ the mobility of electrons in the channel, and $L$ and $W$ the length and width of the channel respectively. $V_{\mathrm{gs}}$ is the gate-source voltage and $V_{\mathrm{d}}$ is the drain-source voltage. $V_{t}$ is the so-called threshold voltage of the PRESSFET, which depends on the capacitance $C_{t}$ between the gate and silicon and on the charge in the insulator [5], thus also on the electret charge. Usually the stored charge $Q_{e}$ (per unit area) in the electret foil is located almost at the electret-air interface, yielding the following expression for $V_{t}$ :

$V_{\mathrm{t}}=\varphi_{\mathrm{ms}}+2 \varphi_{\mathrm{F}}-\frac{Q_{\mathrm{ox}}}{C_{\mathrm{t}}}-\frac{Q_{\mathrm{d}}}{C_{\mathrm{t}}}-\frac{Q_{\mathrm{e}}}{C_{\mathrm{a}}}$

with $\varphi_{F}$ the Fermi potential difference between doped bulk silicon and intrinsic silicon and $\varphi_{\mathrm{ms}}$ the metal-silicon Fermi potential difference. The charge $Q_{o x}$ per unit area accounts for the net charge in the silicon dioxide as well as for the charged surface states at the silicon-silicon dioxide interface and the charge $Q_{d}$ per unit area is the depletion charge. Note that the electret charge $Q_{e}$ at the electret-air interface introduces a threshold voltage shift of $-Q_{\mathrm{e}} / C_{\mathrm{n}}$.

Combining eqns. (4) and (5), we obtain the following relation:

$I_{\mathrm{d}}=\mu \frac{W}{L}\left[C_{\mathrm{t}}\left(V_{\mathrm{zs}}+\frac{Q_{\mathrm{e}}}{C_{\mathrm{a}}}-\varphi_{\mathrm{ma}}-2 \varphi_{\mathrm{F}}-0.5 V_{\mathrm{ds}}\right)+Q_{\mathrm{ox}}+Q_{\mathrm{d}}\right] V_{\mathrm{de}}$

In practical situations the thickness of the electret foil is of the order of several microns, while the oxide thickness is generally of the order of onetenth of a micron. Obviously we can say that $C_{\mathrm{ox}} \rightarrow C_{\mathrm{e}}$. Using a reasonable value for $Q_{e}$ of about $-40 \mathrm{nC} / \mathrm{cm}^{2}$ and assuming the air-gap capacitance $C_{\mathrm{a}}$ per unit area to be about $2 \times 10^{-10} \mathrm{~F} / \mathrm{cm}^{2}$, which is the case for an airgap space of about $5 \mu \mathrm{m}$, it appears that the term $Q_{\mathrm{e}} / C_{\mathrm{a}}$ is about $-200 \mathrm{~V}$. This, however, implies that in practical situations all other terms present in eqn. (6), except $Q_{o x}$ and $Q_{d}$, can be neglected. In this case the drain current $I_{\mathrm{a}}$ can be approximated by: 
$I_{\mathrm{d}}=\mu \frac{W}{L}\left[Q_{\mathrm{e}} \frac{C_{\mathrm{e}}}{C_{\mathrm{e}}+C_{\mathrm{a}}}+Q_{\mathrm{ox}}+Q_{\mathrm{d}}\right] V_{\mathrm{ds}}$

Using this result, the differential sensitivity of the sensor, $\delta I_{\mathrm{d}} / \delta P$, can be calculated by combining eqns. (2) and (7), and differentiating $I_{\mathrm{d}}$ with respect to $P$. Assuming $P$ to be zero, and thus $d_{\mathrm{a}}$ and $C_{\mathrm{a}}$ to be $d_{\mathrm{a} o}$ and $C_{\mathrm{ao}}$ respectively, and using eqn. (3), the following expression is found:

$\frac{\delta I_{\mathrm{d}}}{\delta P}=-\mu \frac{W}{L} \frac{Q_{\mathrm{e}} C_{\mathrm{a} 0} C_{\mathrm{e}}}{P_{\mathrm{m}}\left(C_{\mathrm{a} 0}+C_{\mathrm{e}}\right)^{2}} V_{\mathrm{ds}}$

Observing eqn. (8), we see that the pressure sensitivity is inversely proportional to the maximum measurable pressure $P_{m}$. This means that pressure range and pressure sensitivity are related to each other. We also see that the optimum value of the electret capacitance (for optimum pressure sensitivity) is equal to $C_{\mathrm{ao}}$.

As mentioned above, the addition of an electret manifests itself as an extra bias voltage. Removing the electret charge, a voltage of about $200 \mathrm{~V}$ between gate and source is required to achieve the same sensitivity.

\section{PRESSFET processing}

Much is already known concerning the realization of three-dimensional structures combined with a MOS-detection circuit. There is, however, not a uniform approach to the realization of such structures, because of their specific requirements that have to be taken into account [6-9]. This is also the case with the PRESSFET structure, and therefore its processing will be discussed step by step.

Electrets are mainly realized by using commercially available Teflon foil, because of its very large resistivity of about $10^{21} \Omega \mathrm{cm}$; the foil is deposited on a rigid backplate and afterwards charged. In our case this backplate is a silicon wafer that has already passed several NMOS processing steps and has a top-layer of silicon dioxide. The silicon wafer should be provided with holes from back to front, which later on, for instance, will be used as inlets for a reference pressure to the air gap. The bonding-strength of Teflon to silicon dioxide appears to deteriorate when the wafer is exposed to the etching fluids generally used for the realization of holes going from back to front in a silicon wafer. This means that the creation of holes has to precede the Teflon deposition. On the other hand, it should be mentioned that the deposition of a Teflon layer on a wafer provided with holes results in cracking and damaging of this layer over a considerably large area around every hole. This rather paradoxical problem can only be solved by determining the appropriate sequence of processing steps, which will now be discussed briefly. 


\subsection{The NMOS process}

We use a CVD process to form doped and undoped silicon dioxide layers, which can be patterned and afterwards used as diffusion sources to dope the silicon wafer.

We start from p-type silicon wafers with an initial boron concentration of about $2 \times 10^{15} / \mathrm{cm}^{3}$ and a resistivity of about $10 \Omega \mathrm{cm}$. The front and back of the wafer are provided with a boron-doped and an undoped oxide layer respectively. The boron-doped layer is used to enhance the boron doping of the wafer, avoiding the occurrence of parasitic channels. The undoped layer on the back is used as an etching mask for the realization of holes through the silicon wafer by means of anisotropic etching from back to front in a later stage of the process. The required alignment marks on both sides, to control the exact location of the holes, are realized by photolithography using a mechanical holder containing two identical masks, aligned with respect to each other.

On the patterned boron-doped layer a phosphorus-doped oxide layer is deposited to dope the source and drain regions, and patterned as schematically drawn in Fig. 2(a).

(a)

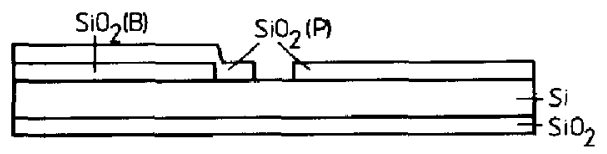

(b)

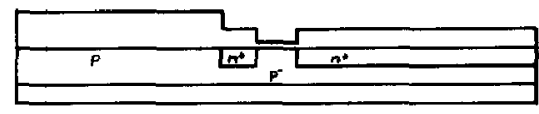

(c)

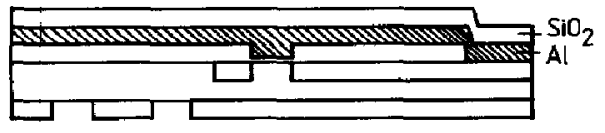

(d)
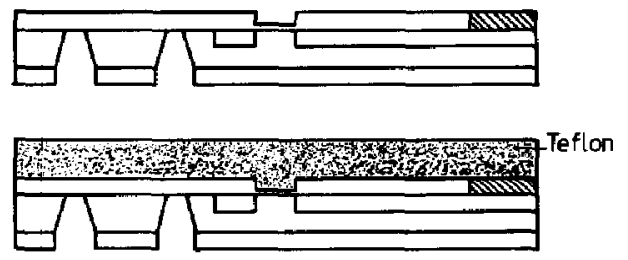

(e)

(f)

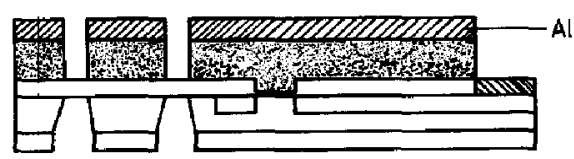

(g)

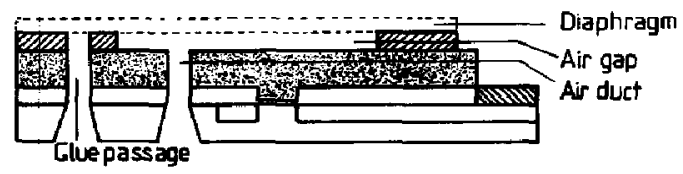

Fig. 2. Processing steps of the PRESSFET. 
Oxidation of the gate-region and diffusion of boron and phosphorus from the oxide into the silicon are performed at $1150{ }^{\circ} \mathrm{C}$, resulting in the structure drawn in Fig. 2(b).

Now the oxide on the back, which will be used as a mask for the etching of holes through the silicon, is patterned and the contact holes on the front for the source, bulk and drain contacts are etched. Then a $1 \mu \mathrm{m}$ thick aluminium layer is evaporated on the front for the realization of the contacts. This aluminium layer is covered with an oxide layer by means of CVD, to protect the front of the wafer during the etching of holes through the silicon, as schematically drawn in Fig. 2(c).

The anisotropic etching of holes through the silicon is then performed by means of an ethylenediamine-pyrocatechol-pyrazine-water mixture, kept at a constant temperature of $90^{\circ} \mathrm{C}$. After the silicon etching has been terminated, the oxide layer on top of the aluminium is removed and the aluminium layer itself is patterned in order to realize the contacts. The structure is schematically drawn in Fig. 2(d).

The holes, etched through the silicon wafer, are still closed by the oxide that has been used to dope the silicon wafer. This, however, means that on this structure the Teflon foil can be deposited without being cracked or damaged.

\subsection{Teflon deposition and etching}

To realize electrets, we decided to apply commercially available materials generally in use for this purpose. From the literature it is known that especially Teflon-FEP (a registered trademark of Du Pont and available in $25 \mu \mathrm{m}$ and $12.5 \mu \mathrm{m}$ thick foils) can be applied as an electret material [10]. These foils are deposited on a silicon wafer by a heat-sealing technique (see Fig. 2(e)).

It will be clear that this Teflon layer has to be patterned, for instance above the source, bulk and drain contacts. We have found that this very inert layer can only be etched by using a reactive ion etcher containing an $\mathrm{O}_{2}$ plasma, as described by Pederson [11]. Aluminium, evaporated on top of the Teflon, can be used as a masking material during the dry etching. In Fig. 2(f) we have drawn the structure after etching the Teflon.

After Teflon etching the aluminium mask is removed, except for a ring around the gate of the NMOS structure. In this way we can realize an air gap between the Teflon and the diaphragm, whose zero-pressure space (written as $d_{\mathrm{a} 0}$ in Section 2) is thus determined by the thickness of the original aluminium mask. Next the holes through the wafer, which are still closed by a thin oxide layer, as drawn in Fig. 2(f), have to be opened. Due to the fact that etchants for $\mathrm{SiO}_{2}$ are less agressive with respect to the Teflon bonding than those used for the anisotropic etching of silicon, and the required etch-time is much shorter, the Teflon attachment remains undamaged if the oxide layer is now etched. We thus obtain the structure as schematically drawn in Fig. 2(g). 


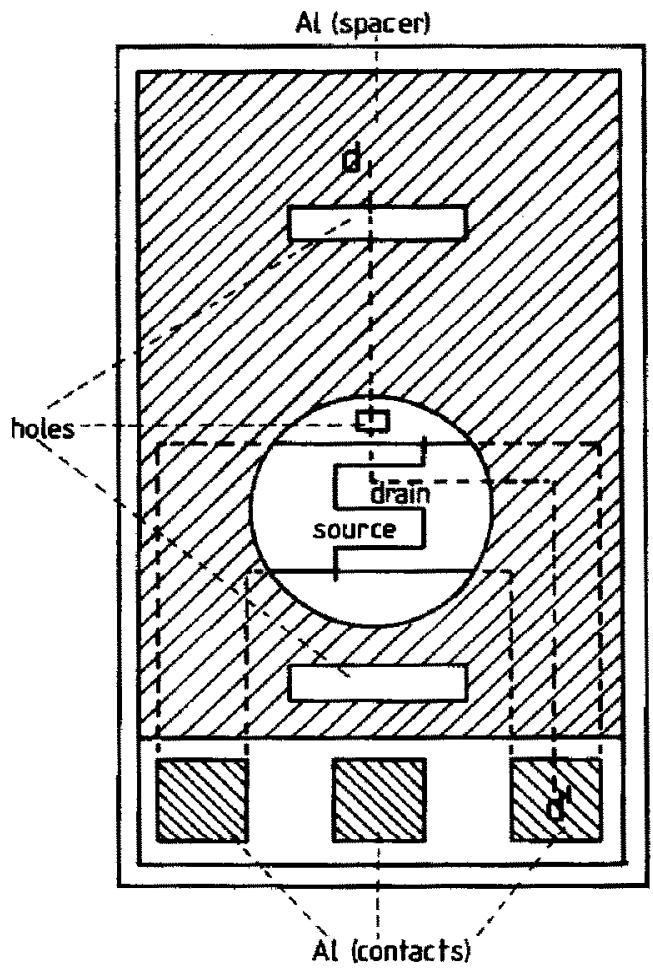

Fig. 3. Top-view of the PRESSFET before diaphragm attachment.

A top-view of the structure is drawn in Fig. 3. We observe three holes, of which the smallest one is used as an inlet for the reference pressure, usually the barometric pressure. In this way the PRESSFET can measure pressures relative to the barometric pressure. The two larger holes are used for the admission of bonding glue for attachment of the diaphragm. Before the diaphragm will be assembled, however, the Teflon has to be provided with electric charge.

\subsection{Teflon charging}

Many different methods to form electrets are proposed in the literature $[10,12$ - 16]. The 'corona', the 'liquid contact' and the 'electron beam' methods are well known. The charge-stability of electrets realized by any of these methods appeared to be competitive. The 'electron beam' charging method, however, provides the opportunity to create electrets with a controlled charge distribution over distances less than $100 \mu \mathrm{m}$ [17]. Considering our structure, as drawn in Fig. 3, with a large aluminium area and a restricted Teflon area, we decided to use the 'electron beam' charging method to form electrets.

The electrons of the beam strike the non-metallized surface of the Teflon sample. Due to collisions inside the Teflon, they are slowed down and trapped, forming a negatively-charged electret. It is known that for the 'electron beam' forming method, the electret charge is not located at the 
Teflon surface, but that it is distributed over a depth of several microns [18]. In our case we have found an average penetration depth of $2.5 \mu \mathrm{m}$.

\subsection{Diaphragm attachment}

After the Teflon foil has been charged, the pressure-sensitive diaphragm should be assembled on top of the structure as shown in Fig. 2(g). To conserve the electret charge, we have used a low-temperature and dry deposition method. As a first approach we decided to realize the diaphragm by starting from commercially available aluminium foils with a thickness of 3 or $8 \mu \mathrm{m}$, which are bonded to the MOS structure by using glue. Therefore the silicon body of every device is provided with two holes, which are filled with glue after the diaphragm and FET structure are put together. On-wafer diaphragm attachment is of course desirable, but has up to now not been investigated in detail.

\section{Experimental}

The numerical values of the PRESSFET parameters determining the sensor behaviour are:

Mobility of charge carriers

Width of the channel

Length of the channel

Electret charge per unit area

Net oxide charge

Depletion charge

Zero-pressure air-gap space

Thickness of Teflon foil

Gate-oxide thickness

Relative dielectric constant electret (Teflon)

Relative dielectric constant $\mathrm{SiO}_{2}$

Diaphragm radius

Flexural rigidity ( $3 \mu \mathrm{m}$ thickness)

Flexural rigidity ( $8 \mu \mathrm{m}$ thickness)

Drain-source voltage

Length of the sensor

$\stackrel{\mu}{W}$

$L$

$Q_{e}$

$Q_{\text {ox }}$

$Q_{\mathrm{d}}$

$d_{\mathrm{a} 0}$

$d_{t}$

$d_{\text {ox }}$

$\epsilon_{\mathrm{e}}$

$\epsilon_{\mathrm{ox}}$

$a$

D

$D$

$V_{\text {ds }}$
$600 \mathrm{~cm}^{2} / \mathrm{Vs}$

$800 \mu \mathrm{m}$

$15 \mu \mathrm{m}$

$-30 \mathrm{nC} / \mathrm{cm}^{2}$

$130 \mathrm{nC} / \mathrm{cm}^{2}$

$-15 \mathrm{nC} / \mathrm{cm}^{2}$

$5 \mu \mathrm{m}$

$10 \mu \mathrm{m}$

$70 \mathrm{~nm}$

2

3.9

$250 \mu \mathrm{m}$

$2.5 \times 10^{-7} \mathrm{~N} \mathrm{~m}$

$4.7 \times 10^{-6} \mathrm{~N} \mathrm{~m}$

$1 \mathrm{~V}$

$2 \mathrm{~mm}$

$1 \mathrm{~mm}$

Width of the sensor

Using these values and eqn. (8), the calculated differential pressure sensitivity is $160 \mu \mathrm{A} / 100 \mathrm{~mm} \mathrm{Hg}(12 \mathrm{nA} / \mathrm{Pa})$ and $8.3 \mu \mathrm{A} / 100 \mathrm{~mm} \mathrm{Hg}$ $(0.62 \mathrm{nA} / \mathrm{Pa})$ for 3 and $8 \mu \mathrm{m}$ thick diaphragms respectively. According to eqn. (5), the maximum pressure $P_{\mathrm{m}}$ appears to be $150 \mathrm{~mm} \mathrm{Hg}(20 \mathrm{kPa})$ or $2900 \mathrm{~mm} \mathrm{Hg}(380 \mathrm{kPa}$ ) for 3 and $8 \mu \mathrm{m}$ thick diaphragms respectively.

To measure our devices, we have realized a pressure actuator by using an air-filled metal bellows, which can be expanded or compressed by a socalled linear motor. The pressure in the bellows is sensed by a commercial 
pressure sensor, of which the output signal is fed back to an electronic controller connected to the linear motor. In this way pressures between $-200 \mathrm{~mm} \mathrm{Hg}$ and $+400 \mathrm{~mm} \mathrm{Hg}$ can be generated with an accuracy of $98.5 \%$ for frequencies between 0 and $100 \mathrm{~Hz}$ [19].

Using this pressure actuator the characteristics of our devices have been measured. In Fig. 4 the measured drain current is shown for two typical devices with a $3 \mu \mathrm{m}$ thick diaphragm. We observe a pressure sensitivity of about $30 \mu \mathrm{A} / 100 \mathrm{~mm} \mathrm{Hg}$ for pressures between -100 and $+400 \mathrm{~mm} \mathrm{Hg}$, which is about five times lower than the calculated value mentioned above. Due to the fact that the calculated value of the maximum pressure $\boldsymbol{P}_{\mathrm{m}}$ for devices with a $3 \mu \mathrm{m}$ diaphragm is $150 \mathrm{~mm} \mathrm{Hg}$, we expect a considerable reduction of the pressure sensitivity at this pressure. Only one of the measured devices, as shown in Fig. 4, displays this effect for a pressure of about $250 \mathrm{~mm} \mathrm{Hg}$.

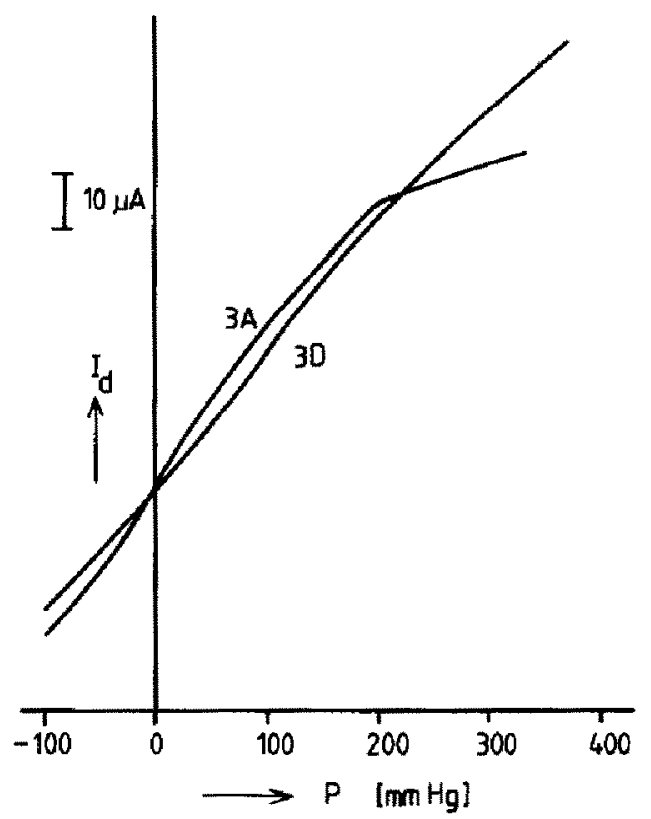

Fig. 4. Measured drain current as a function of pressure for devices with 3 micron thick diaphragm.

Devices with an $8 \mu \mathrm{m}$ diaphragm showed a linear pressure sensitivity of about $3 \mu \mathrm{A} / 100 \mathrm{~mm} \mathrm{Hg}$ in the range $-100 \mathrm{~mm} \mathrm{Hg}$ to $+400 \mathrm{~mm} \mathrm{Hg}$, which is about three times lower than the calculated value.

With respect to the temperature sensitivity of our devices, we have measured that a change of $1^{\circ} \mathrm{C}$ causes a drain current change of $10 \mu \mathrm{A}$. Considering a pressure sensitivity of $30 \mu \mathrm{A} / 100 \mathrm{~mm} \mathrm{Hg}$, we can say that a temperature change of $1^{\circ} \mathrm{C}$ corresponds to a pressure change of $33 \mathrm{~mm} \mathrm{Hg}$. It will be clear that this value is far too high and should be reduced dramatically. 


\section{Conclusions and discussion}

In order to compare our results with those of others, we mention that the drain current for zero pressure is about $3 \mathrm{~mA}$. In that case the measured relative change of the drain current appears to be about $10 \mathrm{~mA} / \mathrm{A} / 100 \mathrm{~mm}$ $\mathrm{Hg}$ and $1 \mathrm{~mA} / \mathrm{A} / 100 \mathrm{~mm} \mathrm{Hg}$ for devices with 3 and $8 \mu \mathrm{m}$ thick diaphragms respectively. For piezoresistive devices with comparable dimensions, the relative sensitivity is about $1 \mathrm{mV} / \mathrm{V} / 100 \mathrm{~mm} \mathrm{Hg}$. Realizing that the theory of the PRESSFET predicts a higher value of the sensitivity, we conclude that its sensitivity is at least comparable with that of other pressure sensors.

Considering the experimental results, we observe a lower pressure sensitivity than expected from theory. We have also observed that the maximum pressure $P_{m}$ that can be measured is higher than expected from the calculated results. These facts are in agreement with each other, as can be seen from eqn. (9). The main reason for the lower value of the pressure sensitivity seems to be a higher value of $\boldsymbol{P}_{m}$ than is calculated from eqn. (5). A reason for this can be the presence of tensile forces in the diaphragm, which reduce its deflection as a function of pressure [20].

The temperature sensitivity is mainly caused by the temperature dependence of the mobility $\mu$, which is known to vary about $0.3 \% /{ }^{\circ} \mathrm{C}$. This, however, means (for a drain current of $3 \mathrm{~mA}$ ) a change of $9 \mu \mathrm{A} /{ }^{\circ} \mathrm{C}$. To reduce this temperature sensitivity compensation circuitry will be necessary. At the same time it should be noted that an increase of the pressure sensitivity, for instance by reducing the tensile forces in the diaphragm, also reduces the relative influence of temperature changes.

An interesting aspect of the PRESSFET is the fact that we have integrated a self-biased pressure-sensitive capacitor and a single MOS transistor as the detection circuitry. In this way we avoid the need for an external bias voltage, which fact might be of importance for biomedical applications.

Several problems that have to be solved are the complexity of the fabrication process, the lower sensitivity than expected from theory and the high temperature sensitivity. The primary aim of the research described in this paper was to verify the validity of the sensor principle and the associated theory. Further research will be focused on solving the problems mentioned above.

\section{Acknowledgments}

The authors wish to thank Prof. O. W. Memelink, Dr. H. Wallinga and Ir. A. J. Sprenkels for many helpful discussions and A. J. Verloop for this technical support.

\section{References}

$1 \mathrm{~J}$. A. Voorthuyzen and P. Bergueld, Theoretical considerations in the design of integrated semiconductor sensors applying electrets, IEEE Trans. Electron Devices, ED-32 (7) 1185 - 1190 . 
2 A. J. Sprenkels, J. A. Voorthuyzen and P. Bergveld, A theoretical analysis of the electret air gap field effect structure for sensor applications, Sensors and Actuators, 9 (1983) $59-72$.

3 S. Timoshenko and S. Woinowaki-Krieger, Theory of Plates and Shells, MeGraw-Hill, New York, 2nd edn., 1959.

4 R. S. Muller and T. I. Kamins, Device Electronics for Integrated Circuits, Wiley, New York, 1977.

5 D. Frohman-Bentchkowsky, The metal-nitride-oxide-silicon (MNOS) transistor characteristics and applications, Proc. IEEE, 58 (8) (1970) $1207-1219$.

6 K. E. Petersen, A. Shartel and N. F. Raley, Mícromechanical accelerometer integrated with MOS detection circuitry, IEEE Trans. Electron Devices, ED-29 (1) (1982) $23-27$.

7 P.-L. Chen, R. S. Muller, R. D. Jolly, G. L. Halac, R. M. White, A. P. Andrews, T. C. Lim and $M$. E. Motamedi, Integrated silicon microbeam PI-FET accelerometer, IEEE Trans. Electron Devices, ED-29 (1) (1982) 27 - 33.

$8 \mathrm{H}$. Tanigawa, T. Ishihara, M. Hirata and K. Suzuki, MOS integrated silicon pressure sensor, IEEE Trans. Electron Devices, ED-32 (7) (1985) 1191 - 1195.

9 J. Neumeister, G. Schuster and W. von Munch, A silicon pressure sensor using MOS ring oscillators, Sensors and Actuators, 7 (1985) $167-176$.

10 G. M. Sessler (ed.), Topics in Applied Physics, Vol. 33, Electrets, Springer-Verlag, Berlin, 1980.

11 L. A. Pederson, Structural composition of polymers relative to their plasma etch characteristics, J. Electrochem. Soc., I 29 (1)(1982) 205 - 208.

12 R. A. Moreno and B. Gross, Measurement of potential buildup and decay, surface charge density, and charging currents of corona-charged polymer foil electrets, $J$. Appl. Phys., 47 (8) (1976) 3397 - 3402.

13 G. M. Sessler and J. E. West, Charging of polymer foils with monoenergetic lowenergy electron beams, Appl. Phys. Lett., 17 (12) (1970) 507 - 509.

14 G. M. Sessler and J. E. West, Production of high quasi-permanent charge densities on polymer foils by application of breakdown fields, $J$. Appl. Phys., 43 (3) (1972) 922 926.

15 G. M. Sessler and J. E. West, Electrets formed by low-energy electron injection, J. Electrostatics, 1 (1975) $111 \cdot 123$

16 P. W. Chudleigh, R. E. Collins and G. D. Hancock, Stability of liquid charged electrets, Appl. Phys. Lett., 23 (5) (1973) 211 - 212.

$17 \mathrm{~J}$. Feder, Storage and examination of high-resolution charge images in Teflon foils, J. Appl. Phys., 47 (5) (1976) 1741 - 1745.

18 B. Gross, G. M. Sessler and J. E. West, Location of charge centroid in electron-beamcharged polymer films, J. Appl. Phys., 48 (10) (1977) 4303 - 4306.

19 J. A. Voorthuyzen, The PRESSFET: an integrated electret-MOSFET structure for application as a catheter tip blood-pressure sensor, Ph.D. Thesis, Twente University of Technology, 1986.

20 J. A. Voorthuyzen and P. Bergveld, The influence of tensile forces on the deflection of circular diaphragms in pressure sensors, Sensors and Actuators, 6 (1984) $201-213$.

\section{Biographies}

Johannes Arie Voorthuyzen received the M.S. degree in electrical engineering from the Delft University of Technology, Delft, The Netherlands, in 1982. In the same year he joined the Bio-Information Group, Department of Electrical Engineering, Twente University of Technology, Enschede, The Netherlands, where he received his Ph.D. degree in 1986. His current research is focused on sensors for biomedical applications. 
Piet Bergueld received the M.S. degree in electrical engineering from Eindhoven University of Technology, Eindhoven, The Netherlands, in 1965, and the Ph.D. degree from Twente University, Enschede, the Netherlands, in 1973. In 1984 he was appointed as full professor in biosensor technology at the Twente University of Technology. He is chairman of the Research Unit Sensors and Actuators of this University. He is involved in research on electronic measuring and stimulating methods in physiological systems with special attention for in vivo biosensors. He lectures on biomedical instrumentation for graduate students. 